\title{
Satisfação Sexual entre Homens Idosos Usuários da Atenção Primária'
}

\section{Sexual Satisfaction Among Older Men Assisted by the Brazilian Primary Care}

\author{
Viviane Xavier de Lima e Silva \\ Mestre em Saúde Pública. Médica de Família. \\ Endereço: Rua Mardônio de Albuquerque Nascimento, 119 , Várzea, \\ CEP 50741-380, Recife, PE, Brasil. \\ E-mail: doc_vivi®yahoo.com.br
}

Ana Paula de Oliveira Marques

Doutora em Nutrição. Professora Adjunta do Departamento de Medicina Social da Universidade Federal de Pernambuco.

Endereço: Av. Professor Moraes Rego, s/n, Cidade Universitária, CEP 50739 -970, Recife, PE, Brasil.

E-mail: marquesapøhotmail.com

\section{Jorge Lyra}

Doutor em Saúde Pública. Professor Adjunto do Departamento de Psicologia da Universidade Federal de Pernambuco.

Endereço: Av. Professor Moraes Rego, s/n, Cidade Universitária, CEP 50739-970, Recife, PE, Brasil.

E-mail: jorgelyraळpapai.org.br

\section{Benedito Medrado}

Doutor em Psicologia Social. Professor Adjunto do Departamento de Psicologia da Universidade Federal de Pernambuco.

Endereço: Av. Professor Moraes Rego, s/n, Cidade Universitária, CEP 50739-970, Recife, PE, Brasil.

E-mail: beneditomedradoळgmail.com

\section{Márcia Carréra Campos Leal}

Doutora em Odontologia. Professora Associada do Departamento de Medicina Social da Universidade Federal de Pernambuco.

Endereço: Av. Professor Moraes Rego, s/n, Cidade Universitária, CEP 50739-970, Recife, PE, Brasil.

E-mail: marciacarrera®hotmail.com

\section{Maria Cristina Falcão Raposo}

Doutora em Economia. Professora Adjunta do Departamento de Estatística da Universidade Federal de Pernambuco.

Endereço: Av. Professor Moraes Rego, s/n, Cidade Universitária, CEP 50739-970, Recife, PE, Brasil.

E-mail: cristinaœde.ufpe.br

I Artigo baseado na dissertação de Mestrado em Saúde Coletiva intitulada Até o apagar da velha chama: satisfação sexual entre homens idosos cadastrados no Programa Saúde da Família apresentada ao Programa Integrado de Pós-Graduação em Saúde Coletiva, Departamento de Medicina Social - UFPE, 2008.

Pesquisa com financiamento da CAPES.

\section{Resumo}

Objetivo: Investigar a satisfação sexual entre homens idosos usuários da Estratégia Saúde da Família do Recife. Procedimentos metodológicos: Mediante entrevistas domiciliares face a face, foram estudados 245 homens de 60 a 95 anos, por meio de questionário semiestruturado, anônimo e prétestado. Resultados: A maior parte dos entrevistados compôs-se de pardos (51,8\%), católicos $(67,2 \%)$, com renda familiar de até dois salários mínimos (71,1\%) e média de 3,5 anos de estudo. Quase metade classifica sua saúde como regular. Pouco mais de $83 \%$ residem com uma companheira e $89,7 \%$ destes consideram esse relacionamento como bom ou ótimo. Setenta e três por cento afirmam permanecer sexualmente ativos, sendo os que possuem até 70 anos e que coabitam com uma companheira os de maior frequência sexual. Foi observada associação estatisticamente significativa entre a satisfação sexual atual e a idade, a saúde autopercebida, a satisfação sexual antes dos 60 anos e a frequência sexual. Conclusão: A sexualidade continua presente na vida dos homens maiores de 60 anos. Não se pode minimizar o papel da cultura na qual estão imersos os entrevistados sobre as questões da masculinidade, da velhice e da sexualidade. A vivência da sexualidade e a interpretação dessas experiências por esses homens têm um caráter plural e assim devem ser encaradas pela sociedade e pelas equipes de saúde da família.

Palavras-chave: Sexualidade; Envelhecimento; Homens; Masculinidade; Saúde da família; Atenção primária. 


\section{Abstract}

Objective: Investigate the sexual satisfaction among older men assisted by the Brazilian family health care strategy in city of Recife. Methodological procedures: A sample of 245 men between 60 and 95 years, assisted by the family health care providers, was face-to-face interviewed, with semi-structured, anonymous, standardized questionnaires. Results: The most were non-white (78\%), catholic $(67,2 \%)$, with median of 3,5 years of study and low economic status. Almost a half perceives their health as regular. $83,3 \%$ have a spouse and the most $(89,7 \%)$ classifies this relationship as good or very good. $73 \%$ are sexually active, particularly if they have a spouse or are less 70 years old. 18,1\% were sexually unsatisfied, 32,7\% were indifferent and 49,1\% were satisfied. Statically significant association was observed between the sexual satisfaction and age, perceived health status, past sexual satisfaction and sexual frequency. Conclusions: Independent of the way, sexuality continues present in life of aged men. These men's culture patterns of masculinity, aging and sexuality play an important role in sexual questions. The sexuality and how men interpret these situations have a multiple character and must be faced by society and by Family health care providers as that.

Keywords: Sexuality; Aging; Male; Masculinity; Family Health Care; Primary Care.

\section{Introdução}

Em 1920, as pessoas com 6o anos ou mais representavam apenas $4 \%$ da população brasileira. Em 50 anos, esse percentual aumentou apenas 1\% (5\% da população em 1970). Vinte anos depois, os idosos já representavam $8 \%$ da população brasileira e, em 2005, aproximadamente 10\% (IBGE, 1991; 2006). Estima-se que, em 2050, uma em cada cinco pessoas na população mundial terá mais de 60 anos, sendo essa proporção, nos países industrializados, de uma para cada três pessoas (Pereira e col., 2003).

Segundo a Política Nacional de Saúde do Idoso, o cuidado com a pessoa idosa deve ser baseado na família e na atenção primária à saúde, por meio das Unidades Básicas de Saúde, em especial daquelas sob a Estratégia Saúde da Família, que devem representar para o idoso, idealmente, o vínculo com o sistema de saúde (Silvestre e Costa Neto, 2003).

A ideia da vivência da sexualidade na velhice como benéfica para a saúde, especialmente se associada à qualidade de vida, é frequente na literatura gerontológica (Penteado e col., 2004; Ribeiro e Santos, 2005; Vaz e Nodin, 2005; De Lorenzi e Saciloto, 2006). Porém, a sexualidade na velhice ainda é um tema pouco explorado, até mesmo na prática clínica. Em estudo que investigou o comportamento e a função sexual de 3005 norte-americanos com idade entre 57 e 85 anos, apenas $38 \%$ dos homens e $22 \%$ das mulheres afirmaram ter discutido questões de sua sexualidade com um médico desde os seus 50 anos (Lindau e col., 2007). Em outro trabalho, foi percebido que grande parte dos médicos generalistas não inclui a sexualidade dos pacientes de mais de 50 anos na sua investigação (Gott e col., 2004). Para a Estratégia Saúde da Família, que deve trabalhar com a lógica da prevenção e da promoção da saúde, essa informação é fundamental para o cuidado integral dos cidadãos idosos.

No relatório final da pesquisa "Como estão as relações de gênero no PSF?”, realizada no âmbito da Estratégia Saúde da Família do Recife, sequer há menção da sexualidade das pessoas idosas no capítulo que trata desse tema. Apesar de o enfoque deste estudo ser qualitativo e visar outros objetivos, esse relatório permitiu demonstrar também a menor frequência dos homens às unidades de saúde da 
família, fazendo que se tenha ainda menos informação sobre esses indivíduos (Recife, 2004). Tal dado evidencia a invisibilidade dos homens no cenário da atenção primária, consequentemente, uma maior dificuldade em construir respostas às suas demandas e necessidades no contexto da Estratégia Saúde da Família, atual porta preferencial de entrada no sistema público de saúde (Couto e col., 2010).

Além disso, num cenário de aumento da infecção pelo HIV em maiores de 50 anos (Brasil, 2007), reforça-se o viés moralista que tende a considerar os idosos como população não sexualmente ativa, ou como, na melhor das hipóteses, sempre e inexoravelmente vinculado a um/a único/a parceiro/a. A importância de implantar ações de educação e proteção em relação à sexualidade dos idosos, como se faz com os adolescentes e adultos jovens, ainda está subestimada. Assim, em linhas gerais, essa enviesada leitura sobre a sexualidade idosa tende a promover uma prática também enviesada e certamente parcial, que desconsidera o idoso como sujeito no campo dos direitos sexuais e reprodutivos.

Portanto, estudos que abordem questões sobre a sexualidade masculina em idosos poderão auxiliar gestores no planejamento de ações nas questões ligadas à saúde e à sexualidade no lócus da própria Estratégia Saúde da Família, podendo também ser úteis aos profissionais que atuam diretamente com a clientela idosa, gerando reflexões sobre seus conceitos e suas práticas. Ademais, voltar o olhar para a sexualidade após os 60 anos sugere visibilidade à temática, que, de uma forma geral, é negada pela nossa cultura, embora o conceito de envelhecimento seja algo complexo e heterogêneo. Dessa forma, o presente trabalho buscou investigar a satisfação sexual entre homens idosos, usuários (e potenciais usuários) das ações da Estratégia de Saúde da Família do Recife.

\section{Procedimentos Metodológicos}

O tipo de estudo caracteriza-se como quantitativo e de corte transversal. A cidade do Recife encontra-se dividida em seis Regiões Político-Administrativas (RPA), cada uma subdividida em três microrregiões. Considerando a facilidade de acesso, em virtude de parceria ensino-serviço já existente entre a Univer- sidade Federal de Pernambuco (UFPE) e a Secretaria Municipal de Saúde, foi adotada como lócus privilegiado de investigação a RPA 4, mais especificamente a microrregião $4 \cdot 3$, onde se situa o campus da UFPE e seu entorno.

A microrregião 4.3 localiza-se no extremo oeste de Recife, possui uma extensão territorial de 26,4 $\mathrm{Km}^{2}$ e, em 200o, contava com 18.617 domicílios, com uma média de 3,83 moradores em cada. Oitenta e sete por cento dessas residências possuem água encanada, 98\% dispõem de instalações sanitárias e em igual percentual delas é realizada coleta de lixo (Recife, 2005). Possui sete Unidades de Saúde da Família, que comportam 14 equipes (Recife, 2007), e uma área adscrita ao Programa Agentes Comunitários de Saúde (PACS), vinculado a uma unidade básica, o que faz que grande parte do território dessa microrregião esteja adscrito na atenção primária.

0 tamanho da amostra foi definido considerando uma prevalência esperada do evento investigado de $50 \%$. Esse valor foi determinado por tratar-se de um estudo em que a variável dependente e a maioria das variáveis independentes têm prevalência desconhecida na população de origem. Definiu-se o poder do estudo em $80 \%$, a precisão de $5 \%$, um erro alfa de $5 \%$ e um acréscimo de 10\% no número de participantes da amostra, visando reduzir perdas.

Solicitou-se aos ACS das unidades de saúde da família (USF) da microrregião estudada uma lista com nomes e endereços de todos os homens residentes em suas microáreas, que atendessem aos critérios de inclusão na pesquisa. A partir dessas listas, os possíveis sujeitos foram selecionados por sorteio, de modo a compor uma amostra sistemática. Para o número de indivíduos participantes em cada USF, foi mantida a proporcionalidade com a sua população de homens idosos elegíveis. Os critérios de inclusão utilizados foram idade mínima de 60 anos, sexo masculino, residência na área de estudo, não apresentar enfermidade ou agravo à saúde que trouxesse comprometimentos em termos de comunicação e concordância em participar da pesquisa, após orientação sobre seus objetivos e do compromisso da manutenção do anonimato, com assinatura ou impressão digital em termo de consentimento livre e esclarecido. Cumprindo esses pressupostos, foram entrevistados 245 indivíduos. A taxa de recusas foi 
inferior a $5 \%$.

A coleta de informações para o estudo foi realizada mediante aplicação de questionário semiestruturado, anônimo, com questões pré-testadas, por meio de entrevistas domiciliares, em local privativo, com os idosos selecionados, realizadas por pesquisadores do sexo masculino devidamente treinados, entre os meses de junho e agosto de 2007. Em alguns casos, pelo difícil acesso a algumas moradias, foi necessário contar com o agente de saúde para guiar a visita, mas teve-se o cuidado de ele não participar da entrevista. A elaboração do instrumento de coleta de dados foi baseada em protocolos utilizados pela Pesquisa sobre comportamento sexual e percepções da população brasileira sobre HIV/AIDS, realizada pela Coordenação Nacional de DST/AIDS do Ministério da Saúde; pelo Estudo sobre comportamentos, atitudes e práticas de risco e de prevenção à AIDS entre homens que fazem sexo com homens no Recife, realizado pelo Centro de Pesquisa Aggeu Magalhães e pelo Modern Maturity Sexuality Study, realizado pela AARP - Atlanta/EUA. Durante o projeto piloto, realizado em área com características sociodemográficas semelhantes, foram realizadas as adequações na linguagem do instrumento. Não foi utilizado um formulário autoaplicado porque isso exigiria uma escolarização e capacidade de compreensão de leitura geralmente não encontrada em populações empobrecidas e idosas.

Para análise das informações criou-se um banco de dados, com dupla digitação e validação posterior, no software Epi-Info, versão 6.o4. Posteriormente, os dados foram exportados para o programa Statistical Package for Social Sciences (SPSS) for Windows, versão 10.o. A significância da associação da satisfação sexual com as demais variáveis explicativas foi avaliada por meio do teste Qui-Quadrado de Pearson. Para isso, as variáveis categóricas foram transformadas em variáveis "dummy”. Para verificar a significância estatística da diferença de médias, utilizou-se o teste t de student.

Para explicar o grau de satisfação com a vida sexual atual dos idosos, foi proposto um modelo multinomial logístico, sendo calculados os odds ratios (OR) para cada nível das variáveis explicativas, com intervalo de $95 \%$ de confiança.

0 presente estudo atende às recomendações da resolução $n^{0} .196$ de 10 de outubro de 1996, sobre pesquisa científica desenvolvida com seres humanos. Sua realização foi autorizada pelo Comitê de Ética em Pesquisa do Centro de Ciências da Saúde (CCS) da UFPE, conforme parecer $n^{0}$. 101/o7.

\section{Resultados}

A idade dos sujeitos variou de 60 a 95 anos, com média de 69 anos, e a escolaridade variou de o a 15 anos de estudo, com média de 3,5 anos, sendo 30\% analfabetos. A maioria é parda ( $51,8 \%$ ), seguida dos que se classificam como brancos (22\%) e negros (21,6\%). Aproximadamente $17 \%$ referiram não ter afiliação religiosa no momento da entrevista. Entre os que têm, $67,2 \%$ são católicos e $31,8 \%$ são evangélicos. Porém, cerca de $23 \%$ não frequentam atividades religiosas e, entre os que o fazem, $38 \%$ com frequência inferior a uma vez por mês. No que diz respeito à coabitação no mesmo domicílio, 83,3\% residem com a companheira, $65,3 \%$ com filhos e $33,1 \%$ com netos. Nenhum entrevistado afirmou residir com um companheiro do sexo masculino. Em relação à renda, $54 \%$ ganham até um salário mínimo e 71,1\% até dois salários mínimos. Destaca-se que $67 \%$ dos entrevistados representam a única fonte de renda da família.

Cerca de $49 \%$ consideram sua saúde como regular, 27,3\% consideram-na boa e 6,1\% ótima. Para os que têm companheira, também se observou sua satisfação com esse relacionamento afetivo (89,7\% consideram-no bom ou ótimo).

A idade média da primeira relação sexual foi de 15,8 anos, e a maioria considera que sua vida sexual antes dos 60 anos foi satisfatória $(36,8 \%)$ ou muito satisfatória (47,9\%). Numa escala de um a cinco pontos, onde um representava muito insatisfeito e cinco representava muito satisfeito, a média obtida foi de 4,3 pontos. Considerando os 12 meses anteriores à entrevista, $73 \%$ afirmam permanecer sexualmente ativos, com média de 1,4 parceiros. A frequência de atividade sexual foi maior entre os que têm até 70 anos e entre os que têm uma companheira. Apenas $6,4 \%$ admitiram a prática atual da masturbação, sendo essa prática mais frequente entre os maiores de 70 anos ( $8 \%$ ) e entre os que não coabitam com uma companheira (10,3\%). 
Com relação à satisfação com a vida sexual atual, 18,1\% julgam-se insatisfeitos, 32,7\% são indiferentes e $49,1 \%$ consideram-se satisfeitos. Numa escala de um a cinco pontos, onde um representava muito insatisfeito e cinco representava muito satisfeito, a média obtida foi de 3,4 pontos (Tabela 1).

Tabela I - Proporção (\%) de homens idosos segundo satisfação sexual e grupo etário, vive com companheira e valor médio do grau de satisfação*. Programa Saúde da Família. Recife/PE, 2008

\begin{tabular}{lcccc} 
& Satisfeito (\%) & Indiferente (\%) & Insatisfeito (\%) & Média \\
Total da amostra & 49,1 & 32,7 & 18,1 & 3,4 \\
Até 70 anos & 53,4 & 34,2 & 12,3 & 3,5 \\
Mais de 70 anos & 41,3 & 30,0 & 28,8 & 3,2 \\
Com companheira & 50,0 & 33,5 & 16,5 & 3,4 \\
Sem companheira & 44,7 & 28,9 & 26,3 & 3,2 \\
\hline
\end{tabular}

*escala entre I e 5

Dentre as opções oferecidas para o que ajudaria a aumentar a satisfação sexual, as mais citadas pelos entrevistados foram: melhoria na própria saúde (73,5\%), melhor situação financeira $(56,7 \%)$, melhoria na saúde da parceira (53,9\%) e mais atitudes positivas da família ou da sociedade $(51,4 \%)$.

No que diz respeito às variáveis independentes, foi observada associação significativa na análise bivariada entre o grau de satisfação dos idosos com sua vida sexual atual e a idade, saúde autopercebida, satisfação com a vida sexual antes dos 60 anos e a frequência sexual atual (Tabela 2).

Para tentar explicar o grau de satisfação dos idosos com a vida sexual atual pelos fatores considerados neste estudo, propõe-se um modelo multinomial logístico, em que a categoria "satisfeito" foi utilizada como categoria de referência. Analisando as razões de chances (Tabela 3), vemos que idosos maiores de 70 anos têm 1,7 vezes mais chances de estarem sexualmente insatisfeitos do que idosos mais jovens. Os idosos insatisfeitos com a sua saúde têm 1,7 vezes mais chances de também estarem insatisfeitos com sua vida sexual em comparação com aqueles que se dizem satisfeitos com a sua saúde.
Tabela 2 - Variáveis associadas à satisfação sexual dos idosos. Programa Saúde da Família. Recife/PE, 2008. Satisfação sexual: Insatisfeito $(n=39)$; Indiferente $(n=73)$; Satisfeito $(n=I I)$

\begin{tabular}{lccc} 
Variáveis independentes & $N$ & $\chi 2$ & $P$ \\
Idade & & 9,53 & 0,008 \\
Até 70 anos & 146 & & \\
Mais de 70 anos & 80 & & \\
Saúde autopercebida & & 19,06 & $<0,001$ \\
Não saudável & 36 & & \\
Indiferente & 115 & & \\
Saudável & 75 & & \\
Satisfação sexual antes dos 60 anos & & 18,87 & $<0,001$ \\
Insatisfeito & 9 & & \\
Indiferente & 26 & & \\
Satisfeito & 185 & & \\
Frequência sexual atual & & 38,59 & $<0,001$ \\
Menos de uma vez por mês & 65 & & \\
Uma ou duas vezes por mês & 81 & & \\
Mais de duas vezes por mês & 77 & & \\
\hline
\end{tabular}


Tabela 3 - Modelo Multinomial Logístico: Odds Ratio e Intervalos de Confiança

\begin{tabular}{|c|c|c|c|c|}
\hline \multirow[b]{2}{*}{ Variável } & \multicolumn{2}{|c|}{ Insatisfeito } & \multicolumn{2}{|c|}{ Indiferente } \\
\hline & OR & IC $95 \%$ & OR & IC $95 \%$ \\
\hline \multicolumn{5}{|l|}{ Idade } \\
\hline Até 70 anos & 1 & & 1 & \\
\hline Mais de 70 anos & 1,765 & $0,757-4,117$ & 0,830 & $0,416-1,659$ \\
\hline \multicolumn{5}{|l|}{ Saúde autopercebida } \\
\hline Saudável & 1 & & 1 & \\
\hline Indiferente & 0,804 & $0,299-2,159$ & 4,308 & $1,974-9,402$ \\
\hline Não saudável & 1,700 & $0,514-5,624$ & 4,102 & $1,483-11,348$ \\
\hline \multicolumn{5}{|l|}{ Frequência Sexual } \\
\hline Mais de duas vezes por mês & 1 & & 1 & \\
\hline Uma ou duas vezes por mês & 4,331 & $1,108-16,923$ & 1,405 & $0,684-2,884$ \\
\hline Menos de uma vez por mês & 18,516 & $4,775-71,793$ & 1,167 & $0,489-2,783$ \\
\hline
\end{tabular}

Para a frequência sexual, idosos com menos de uma relação por mês têm 18,5 vezes mais chances de estarem sexualmente insatisfeitos quando comparados aos com mais de duas relações. Essa razão de chances cai para 4,3 entre os idosos que mantêm uma ou duas relações sexuais por mês, em relação aos que mantêm mais de duas relações.

É importante notar que algumas razões de chances apresentaram intervalos de $95 \%$ de confiança que incluem a unidade, o que sugere a não associação estatística desses fatores. Entretanto, é conveniente lembrar que os intervalos construídos são aproximações e só apresentam boa precisão quando o tamanho da amostra é grande.

\section{Discussão}

Existem alterações fisiológicas no corpo com o passar da idade, mas a maior parte da população não tem conhecimento delas (Lopes e col., 1994). Normalmente essas mudanças são vistas como doenças e, principalmente, como perdas, em especial se o corpo for visto como ferramenta de trabalho. Ainda assim, pode-se considerar que os homens estão razoavelmente satisfeitos com seu estado atual de saúde, pois foram encontrados níveis de satisfação semelhantes aos observados em outros estudos realizados com homens residentes em países economicamente mais desenvolvidos (Bortz e col., 1999; AARP..., 1999), que, de forma geral, dispõem de mais recursos para investimento em saúde, tanto privado quanto público, ainda que esse investimento seja feito de forma particular em cada realidade. Esse achado também está em consonância com pesquisa, em âmbito nacional com base na PNAD, realizada por Pinheiro e colaboradores (2002), na qual $59,1 \%$ dos homens urbanos de 65 anos ou mais classificavam sua saúde atual como regular, ruim ou muito ruim.

Parece que os homens estudados que convivem com uma companheira estão satisfeitos com essa relação. Essa alta satisfação poderia ser devida a uma falha metodológica: de alguma forma, os insatisfeitos não encontraram espaço para se manifestar. Porém, é possível que esse achado seja realmente consistente, visto que o aumento da satisfação conjugal com a idade também pode ser observado na literatura (Beutel e col., 2002).

A idade de iniciação sexual foi semelhante à de outro estudo brasileiro realizado com homen (Abdo, 2004) e menor que a observada para as mulheres em faixas etárias próximas (De Lorenzi e Saciloto, 2006). Aparentemente, a vida sexual pregressa dos idosos investigados foi bastante satisfatória, contudo não se pode descartar possível viés de memória, onde só as experiências mais positivas são lembradas.

A exemplo do observado nesta pesquisa, em 
outros estudos (Bortz e col., 1999; Beutel e col., 2002; Abdo, 2004), a maioria dos homens idosos entrevistados manteve atividade sexual nos últimos 12 meses, confirmando que a velhice assexuada é um mito. 0 percentual de homens que tiveram mais de um/a parceiro/a nos últimos 12 meses $(8,5 \%)$ foi discretamente mais baixo do que o encontrado entre homens de 45 a 65 anos (11,15\%) (Barbosa, 2008). Em culturas diferentes, estudando uma faixa etária discretamente mais jovem, foram encontradas prevalências de duas a dez vezes maiores para a prática da masturbação que a do presente estudo (Bortz e col., 1999; AARP..., 1999). Ao falar sobre o assunto, muitos entrevistados posicionam-se contrários a essa prática, considerando-a justificada apenas quando se é muito jovem ou quando não se é casado. Como a última situação não é frequente entre eles, é possível que a prática da masturbação esteja subestimada.

Os homens estudados parecem razoavelmente satisfeitos com sua vida sexual atual, em especial os que têm até 70 anos. Para efeito de comparação com os achados deste trabalho, alguns resultados de outras pesquisas foram convertidos para a escala utilizada. Dessa forma, os resultados são semelhantes aos encontrados entre idosos alemães na mesma faixa de idade para a satisfação sexual global (Beutel e col., 2002). Quando separados por faixa etária, até 70 anos e maiores de 70 anos, em comparação com idosos norte-americanos, os resultados variam de semelhantes (AARP..., 1999) a melhores (Bortz e col., 1999) para os dois grupos etários. Entre os idosos com companheira, a satisfação foi maior que a encontrada entre idosos portugueses de ambos os sexos e idosas brasileiras (Vasconcelos e col., 2004) e semelhante à encontrada entre homens idosos norteamericanos. Os idosos recifenses sem companheira apresentaram melhor nível de satisfação sexual que norte-americanos com mesmo status marital (AARP..., 1999).

As condições de saúde do próprio idoso ou de sua companheira parecem ser as questões que mais interferem na vida sexual dos entrevistados, visto que quase três quartos deles desejam sua melhoria, como forma de aumentar a satisfação sexual. Num contexto socioeconômico mais favorecido, a melhoria na saúde também aparece em primeiro lugar
(Abdo, 2004). Essa informação reforça a importância da atuação das equipes de saúde da família como promotoras de saúde junto à população estudada.

A associação da satisfação sexual com a idade e com a saúde autopercebida também é observada na literatura (Bortz e col., 1999; Beutel e col., 2002; Ribeiro e Santos, 2005; De Lorenzi e Saciloto, 2006). Como no presente estudo, a associação da satisfação sexual com a frequência sexual apresenta forte correlação para idosos alemães (Beutel e col., 2002). Mais uma vez, fica evidenciada a influência do trabalho em promoção da saúde, realizado pelas equipes de saúde da família, na experiência sexual desses usuários.

A melhoria da situação financeira e das atitudes da sociedade frente à sexualidade dos idosos destaca-se como desejo da maioria dos entrevistados, não verificado em contextos de menor faixa etária (Abdo, 2004) ou melhores condições de vida (AARP..., 1999). Se para esses casos a atuação das equipes de saúde da família não é tão direta, ainda assim esses trabalhadores não deixam de ter um importante papel na construção da cidadania de sua clientela, em especial mediante educação e mobilização social.

\section{Conclusão}

Independente da forma, a sexualidade continua presente na vida dos homens maiores de 60 anos. Entre os que mantêm prática sexual, grande parte relata vivenciá-la de maneira diferente de quando eram mais jovens e essa diferença é majoritariamente vista por eles como uma perda, pois estão menos satisfeitos com o presente do que com a lembrança do passado.

É possível que as alterações provocadas no corpo pelo passar dos anos, não necessariamente por meio de doenças, realmente tragam limitações à vida desses idosos. Porém, existe a possibilidade de que, para além do processo de envelhecimento biológico, o desgaste dos relacionamentos com as parceiras, as dificuldades financeiras, as atribulações da vida familiar e a inserção social desses homens tenham sido responsáveis pela menor satisfação com sua vida sexual atual.

Não se pode deixar de mencionar o papel da cultura na qual estão imersos os entrevistados, 
sobre as questões da masculinidade, da velhice e da sexualidade. 0 modelo de masculinidade é ser um homem jovem, produtivo, bom provedor financeiro, cujo lócus de atuação e socialização é a rua e que, no mundo doméstico, detém o poder de decisão sobre os destinos dos seus dependentes e de quem mais habitar o espaço (Medrado, 1997; Medrado e Lyra, 2002; Lyra, 2008). Esses mesmos padrões culturais, que valorizam a capacidade de produção, associam a velhice à perda de autonomia e de valor social.

Além disso, a sexualidade dos homens idosos parece permeada por um jogo complexo em que, por um lado, estimula-se/exige-se uma prática sexual intensa, associada a uma ideia de "qualidade de vida" ou, mais amplamente, de saúde, ao mesmo tempo em que se mantém um jogo de orientações morais que restrigem, controlam e normatizam práticas sexuais, conduzindo a um modelo heteronormativo hegemônico que indiretamente se inscreve em palavras e práticas nas famílias, nas instituições religiosas e também nas instituições de saúde. Como bem destaca Joan Scott (1990), ninguém reproduz literalmente os modelos culturais de gênero. Portanto, esses padrões culturais hegemônicos, com ideais praticamente intangíveis, mas fortemente exigidos para os homens, como os sujeitos da pesquisa, tendem a produzir frustração e possível baixa autoestima, interferindo também em sua satisfação sexual.

Imersos nesse mesmo universo sociocultural, os formuladores de políticas, gestores e trabalhadores da saúde enfrentam dificuldades para reverem suas atuações. Nas últimas décadas, vem acontecendo uma maior visibilização dos homens na pauta da saúde coletiva, primordialmente por meio das temáticas da saúde sexual e reprodutiva e da violência, ressaltando "o entrelaçamento entre saúde, cidadania e direitos humanos" (Schraiber e col., 2005, p. 8). Porém, um longo caminho ainda é necessário. Especificamente com relação à sexualidade masculina, torna-se fundamental uma discussão sobre a influência do imaginário dessa masculinidade hegemônica sobre os cuidados ou não com a saúde, muito bem exemplificada por Gomes (2003), discorrendo sobre o toque retal na prevenção do câncer de próstata, para que as ações influenciadas por esses sentidos simbólicos possam ser melhor conduzidas.

As equipes de saúde da família parecem não estar preparadas para lidar com essa realidade. Dentre os inúmeros problemas que elas enfrentam, a falta de preparação para a subjetividade das situações, a deficiência de atualização das condutas, a dificuldade de reflexão sobre as práticas e o insuficiente apoio dos gestores, em todos esses níveis, entravam a resolução dos problemas voltados à sexualidade dos homens idosos. A mudança dos padrões culturais opressores deve ser objetivo de todos os envolvidos com as temáticas do envelhecimento e da sexualidade. Conceitos como o do idoso associado à decrepitude, da velhice assexuada, de uma sexualidade correta única, da monogamia presumida, entre outros, são causa de grandes sofrimentos, tanto individuais como coletivos. São necessárias reflexão e ação contínuas, em todas as esferas de atuação, a serviço da construção de um mundo melhor, mais justo e mais feliz, para todos os indivíduos, incluindo os de mais idade.

\section{Referências}

AARP/MODERN MATURITY. Sexuality study. Washington, DC: 1999. Disponível em: <http:// assets.aarp.org/rgcenter/health/mmsexsurvey. pdf >. Acesso em: 15 jan. 2010.

ABDO, C. Estudo da vida sexual do brasileiro. São Paulo: Bregantini, 2004.

BARBOSA, R. M. et al. Comportamento e práticas sexuais de homens e mulheres, Brasil 1998 e 2005. Revista de Saúde Pública, São Paulo, v. 42, supl. 1, 2008.

BEUTEL, M. E et al. Sexual activity, sexual and partnership satisfaction in ageing men - results from a German representative community study. Andrologia, Berlim, v. 34, n. 1, p. 2-8, 2002.

BORTZ, W. M; WALLACE, D. H; WILEY, D. Sexual function in 1202 aging males: differentiating aspects. Journal of Gerontology: Biologycal Sciences, Washington, v. 54, n. 5, p. M237-241, 1999. 
BRASIL. Ministério da Saúde. Datasus. Casos de AIDS identificados no Brasil. Brasília, 2007. Disponível em: <www.aids.gov.br/cgi/deftohtm. exe?tabnet/br.def $>$. Acesso em: 02 dez. 2009.

COUTO, M. T. et al. Men in primary healthcare: discussing (in)visibility based on gender perspectives. Interface - Comunicação, Saúde e Educação, Botucatu, SP, v. 14, n. 33, p. 257-27o, abr./jun. 2010

De LORENZI, D. R. S; SACILOTO, B. Frequência da atividade sexual em mulheres menopausadas. Revista da Associação Medica Brasileira, São Paulo, v. 52 n. 4, p. 256-26o, jul/ago. 2006.

GOMES, R. Sexualidade masculina e saúde do homem: proposta para uma discussão. Ciência $\&$ Saúde Coletiva, Rio de Janeiro, v. 8, n. 3, p. 825$829,2003$.

GOTT, M; HINCHLIFF, S.; GALENA, E. General practitioner attitudes to discussing sexual health issues with older people. Social Sciences and Medicine, Oxford, v. 58, n.11, p. 2093-2103, 2004.

IBGE - INSTITUTO BRASILEIRO DE GEOGRAFIA E ESTATÍSTICA. Estatísticas do século XX. Brasília, DF: IBGE, 1991. Disponível em: http://www.ibge. gov.br/seculoxx/. Acesso em: 02 dez. 2009.

IBGE - INSTITUTO BRASILEIRO DE GEOGRAFIA E ESTATÍSTICA. Pesquisa nacional por amostra de domicílios 2005. Brasília, DF: IBGE, 2006. Disponível em http://www.ibge.gov.br/home/ estatistica/populacao/trabalhoerendimento/ pnad2005/sintesepnad2005.pdf. Acesso em: 2 dez. 2009.

LINDAU, S. T et al. A study of sexuality and health among older adults in the United States. $N$. England Journal of Medicine, Massachusetts, v. 357, n. 8, p.762-774, 2007.

LOPES, G.; TORRES, L. O; MAIA, M. B. Sexualidade e envelhecimento normal. In: CANÇADO, F. A. Noções práticas de geriatria. Belo Horizonte: Coopmed, 1994. p. 402-411.
LYRA, J. Homens, feminismo e direitos reprodutivos no Brasil: uma análise de gênero no campo das políticas públicas (2003-2006). 2008. Tese (Doutorado em Saúde Pública) Departamento de Saúde Coletiva, Centro de Pesquisas Aggeu Magalhães, Fundação Oswaldo Cruz, Recife.

MEDRADO, B. O masculino na mídia: repertórios sobre masculinidade na propaganda televisiva nacional. 1997. Dissertação (Mestrado em Psicologia Social) - Pontifícia Universidade Católica de São Paulo, São Paulo.

MEDRADO, B; LYRA, J. Produzindo sentidos sobre o masculino: da hegemonia à ética da diversidade. In: ADELMAN, M; SILVESTRIN, C. (Orgs.). Curitiba: UFPR, 2002. p. 63-76.

PENTEADO, S. R. L. et al. A. Avaliação da capacidade orgástica em mulheres na pósmenopausa. Revista da Associação Medica Brasileira, São Paulo, v. 5o, n. 4, p. 444-45o, out/ dez. 2004 .

PEREIRA, R. S.; CURIONI, C. C.; VERAS, R. P. Perfil demográfico da população idosa no Brasil e no Rio de Janeiro em 2002. Textos sobre envelhecimento, Rio de Janeiro, v. 6, n. 1, p. 43-59, jan/jul. 2003.

PINHEIRO, R. S. et al. Gênero, morbidade, acesso e utilização de serviços de saúde no Brasil. Ciência \& Saúde Coletiva, Rio de Janeiro, v. 7, n. 4, p. 687707, 2002.

RECIFE. Secretaria Municipal de Saúde. Como estão as desigualdades de gênero no PSF? Relatório final. Recife, 2004. 227 p.

RECIFE. Prefeitura Municipal. Programa das Nações Unidas para o Desenvolvimento - PNUD. Fundação João Pinheiro. Ministério de Integração Nacional. Atlas do desenvolvimento humano no Recife 2005. Recife: Prefeitura do Recife, 2005. RECIFE. Secretaria Municipal de Saúde. Sistema de Informações da Atenção Básica. Recife: SMS, 2007. 
RIBEIRO, J. P; SANTOS, A. Estudo exploratório da relação entre função eréctil, disfunção eréctil e qualidade de vida em homens portugueses saudáveis. Análise Psicológica, Lisboa, v. 23, n. 3 , p. 341-349, julho, 2005.

SCHRAIBER, L. B.; GOMES, R.; COUTO, M. T. Homens e saúde na pauta da Saúde Coletiva. Ciência \& Saúde Coletiva, Rio de Janeiro, v. 1o, n. 1, p. 7-17, 2005.

SILVESTRE, J. A; COSTA NETO, M. M. Abordagem do idoso em programas de saúde da família.

Cadernos de Saúde Pública, Rio de Janeiro, v. 19, n. 3, p. 839-847, maio/jun. 2003.

VASCONCELOS, D et al. A sexualidade no processo do envelhecimento: novas perspectivas comparação transcultural. Estudos de Psicologia, Natal, v. 9, n. 3, p. 413-419, set./dez. 2004.

VAZ, R. A; NODIN, N. A importância do exercício físico nos anos maduros da sexualidade. Análise Psicológica, Lisboa, v. 23, n. 3, p. 329-339, jul. 2005. 